\title{
Ultimate and practical limits of fluid-based mass detection with suspended microchannel resonators
}

\author{
J. L. Arlett and M. L. Roukes ${ }^{\text {a) }}$ \\ Departments of Physics, Applied Physics, and Bioengineering and Kavli Nanoscience Institute, California \\ Institute of Technology, MC 114-36 Pasadena, California 91125, USA
}

(Received 13 March 2010; accepted 4 July 2010; published online 22 October 2010)

\begin{abstract}
Suspended microchannel resonators (SMRs) are an innovative approach to fluid-based microelectromechanical mass sensing that circumvents complete immersion of the sensor. By embedding the fluidics within the device itself, vacuum-based operation of the resonator becomes possible. This enables frequency shift-based mass detection with high quality factors, and hence sensitivity comparable to vacuum-based micromechanical resonators. Here we present a detailed analysis of the sensitivity of these devices, including consideration of fundamental and practical noise limits, and the important role of binding kinetics in sensing. We demonstrate that these devices show significant promise for protein detection. For larger, biologically-important targets such as rare whole virions, the required analysis time to flow sufficient sample through the sensor can become prohibitively long unless large parallel arrays of sensors or preconcentrators are employed. () 2010 American Institute of Physics. [doi:10.1063/1.3475151]
\end{abstract}

\section{INTRODUCTION}

Recently, unprecedented mass sensitivity has been achieved with micromechanical and nanomechanical devices. ${ }^{1-4}$ Underlying these advances is the high susceptibility to added mass that is characteristic of miniature mechanical resonators. Equally important is that microelectromechanical and nanoelectromechanical systems (MEMSs and NEMSs) resonators are capable of attaining very high resonance quality factors $(Q)$. These two attributes, respectively, yield high mass responsivity - that is, frequency shift per added mass increment, and high frequency stabilitynecessary for observing a minute adsorbate-induced frequency shift.

In liquid, micro- and, especially, nanomechanical resonators become heavily fluid-loaded and generally exhibit overdamped response. ${ }^{5,6}$ This strong fluid coupling alters the mass responsivity to some degree but most profoundly impacts the resonator's frequency stability - the fluid damping precipitously suppresses device $Q .{ }^{5}$ For this reason it has not been possible to realize similar advances in mass sensitivity for MEMS and NEMS immersed in liquid as have recently been obtained with vacuum-based devices. This has imposed a significant barrier to biological sensing via mass detection in liquid.

Burg and Manalis ${ }^{7}$ have recently developed an innovative approach to fluid-based mass sensing that circumvents these fundamental limitations. ${ }^{8-10}$ Within a vacuum-based microcantilever structure they embed a microfluidic flow channel, which is subsequently used to transport and present fluid-based analytes to the active vibratory region of this micromechanical mass sensor. Here we analyze practical and fundamental limits to biological mass sensing with such SMRs, focusing on the ultimate sensitivity that is attainable with geometric scaling to nanometer dimensions.

${ }^{a)}$ Electronic mail: roukes@caltech.edu.
Our performance analyses of suspended microchannel resonators (SMRs) are based on two general considerations. First, we draw insights from a number of recent and detailed studies that analyze the kinetics of capture attainable with fluid-immersed sensors. ${ }^{11-14}$ Second, we employ modeling of the ultimate sensitivity of vacuum-based micromechanical and nanomechanical mass sensors (now verified by the aforementioned experiments in multiple laboratories), which has shown that marked improvements are obtainable with scaling downward in size. ${ }^{15}$ We show here that when evaluating suspended microchannel devices, in addition to estimating device responsivity and noise performance, careful consideration of the kinetics of analyte capture is essential. Highlyoptimized devices capable of single-analyte or singlemolecule sensitivity can easily become event limited-that is, at lower concentration levels of biological relevance they may experience a single binding event only extremely infrequently - in the extreme case, only every few days. An example is the recently studied case of an individual nanowire device sensing analytes at $10 \mathrm{fM}$ sensitivity. ${ }^{11}$ In the case of SMRs, an additional limitation arises from the minute volume-handling capacities of individual devices.

SMRs have recently been applied to the measurement of fluid density ${ }^{16,17}$ and more recently to biosensing in liquid. ${ }^{9,10,18-20}$ Underlying these advances is the high quality factors attainable with these fluid-filled devices; values of 15000 have been demonstrated, which are equivalent to those observed when the device is empty. ${ }^{10}$ These results are in sharp contrast with those obtained from fluid-immersed mechanical resonators, where $Q$ 's of order 1-10 are typical. ${ }^{6}$ It is possible to enhance these low $Q$ 's from fluid-immersed devices by operating them using higher-order vibrational modes or stiffer geometries. The resulting higher frequency operation serves to increase the Reynold's number ${ }^{21-23}$ but even with this enhancement, $Q$ 's rise only to values of order 
TABLE I. Physical parameters for prototype Si microchannel resonators. Parameters tabulated are thickness, $t$; width, $w$; length, $\ell$; channel thickness, $t_{c h a n}$, and channel width, $w_{\text {chan }}$ (the latter value is for doubly-clamped beams, for cantilevers, the width of an individual channel is $w_{\text {chan }} / 2$ ), Quality factor, $Q$, frequency in vacuum, $f$; force constant, $K$; mass sensitivity $(1 \mathrm{~Hz}$ bandwidth) in the fundamental limit (for which the dynamic range is determined by thermomechanical noise and the onset of nonlinearity), $\delta \mathrm{m}_{\text {fund }}$ and mass sensitivity (1 Hz bandwidth) in the practical limit (piezoresistive detection and thermoelastic actuation), $\delta \mathrm{m}_{\text {pract }}$. The resonance frequency and spring constant are computed by finite element simulation (COMSOL) and include the effects of mass loading by the fluid (water). These values shown are for devices fabricated from silicon. The quality factors are extrapolated based on measured quality factors of fluid-filled SMRs (Refs. 8 and 10) and their projected scaling based on previous studies of the quality factor of MEMS/NEMS resonators in vacuum (Ref. 25).

\begin{tabular}{|c|c|c|c|c|c|c|c|c|c|c|c|c|c|c|}
\hline \multirow[b]{2}{*}{ No. } & \multirow[b]{2}{*}{$\begin{array}{c}t \\
(\mathrm{~nm})\end{array}$} & \multirow[b]{2}{*}{$\begin{array}{c}w \\
(\mu \mathrm{m})\end{array}$} & \multirow[b]{2}{*}{$\begin{array}{c}\ell \\
(\mu \mathrm{m})\end{array}$} & \multirow[b]{2}{*}{$\begin{array}{l}t_{\text {chan }} \\
(\mathrm{nm})\end{array}$} & \multirow[b]{2}{*}{$\begin{array}{l}w_{\text {chan }} \\
(\mu \mathrm{m})\end{array}$} & \multirow[b]{2}{*}{$Q$} & \multicolumn{4}{|c|}{ Cantilevers } & \multicolumn{4}{|c|}{ Doubly-clamped beams } \\
\hline & & & & & & & $\begin{array}{c}f \\
(\mathrm{MHz})\end{array}$ & $\begin{array}{c}K \\
(\mathrm{~N} / \mathrm{m})\end{array}$ & $\begin{array}{c}\delta \mathrm{m}_{\text {fund }} \\
(\mathrm{ag})\end{array}$ & $\begin{array}{c}\delta \mathrm{m}_{\text {pract }} \\
(\mathrm{ag})\end{array}$ & $\begin{array}{c}f \\
(\mathrm{MHz})\end{array}$ & $\begin{array}{c}K \\
(\mathrm{~N} / \mathrm{m})\end{array}$ & $\begin{array}{c}\delta \mathrm{m}_{\text {fund }} \\
(\mathrm{ag})\end{array}$ & $\begin{array}{c}\delta \mathrm{m}_{\text {pract }} \\
\text { (ag) }\end{array}$ \\
\hline 1 & 7000 & 40 & 200 & 5000 & 20 & 11000 & 0.22 & 46 & 4.6 & 141 & 1.3 & 2730 & 12 & 179 \\
\hline 2 & 3500 & 20 & 100 & 2500 & 10 & 8800 & 0.44 & 23 & 1.2 & 15 & 2.7 & 1360 & 2.9 & 23 \\
\hline 3 & 1400 & 8 & 40 & 1000 & 4 & 7600 & 1.1 & 9.2 & 0.18 & 1.5 & 6.7 & 546 & 0.47 & 2.0 \\
\hline 4 & 700 & 4 & 20 & 500 & 2 & 7200 & 2.2 & 4.6 & 0.046 & 0.27 & 13.5 & 273 & 0.12 & 0.35 \\
\hline 5 & 350 & 2 & 10 & 250 & 1 & 7000 & 4.4 & 2.3 & 0.012 & 0.09 & 27.4 & 137 & 0.029 & 0.11 \\
\hline
\end{tabular}

30. Nonetheless, even such modest improvements can be sufficient to enable useful biosensing applications. ${ }^{22}$

In practical implementations, however, kinetics can become a limiting factor with SMR devices due to practical constraints on the maximum volume of fluid that can flow through a narrow channel at tolerable operating pressures and time intervals. We present here a detailed analysis of the sensitivity of such devices for the detection of biological targets that are both large (e.g., virions) and small (e.g., proteins). In our analyses below we will consider two distinct modes of detection: flow-through and affinity-capture based detection.

\section{OVERVIEW: FLOW-THROUGH AND AFFINITY- CAPTURE MODES OF DETECTION}

Two modalities of operation are possible with SMR devices; namely, flow-through detection in which the target analyte is detected by accurately measuring its mass as it passes through the channel, and affinity-based capture in which target analytes are captured by molecular recognition on a biofunctionalized surface of the device. Flow-through detection obviates the need for expensive reagents and sample preparation that are essential for preparing functionalized surfaces required for specific capture. However, flowthrough detection is only possible for situations where the detector sensitivity is sufficient for single-particle detection, in other words, where the resolution enables distinguishing target particles from other constituents of the sample solution. For the smallest targets, such as individual proteins, this can prove to be challenging - and detection by affinity-based capture may provide a more practical approach.

By contrast, the sensitivity requirements for detection with functionalized capture are less stringent than for flowthrough detection for two reasons: (1) the added mass signal from immobilized target analytes can be averaged over a period of time longer than that for free-particle transit through the microchannel; (2) the functionalization can provide high specificity-of-capture. This can reduce false positives arising from particles of similar mass, which can occur for operations that are based on nonspecific, flow-through detection.

\section{ANALYSIS OF FLOW-THROUGH DETECTION: INDIVIDUAL VIRIONS}

\section{A. Flow-through detection: Device geometry, operating pressure, and sensitivity impose practical limits on analyte flux}

As mentioned, a basic criterion for performing flowthrough detection is that the device should have sufficient sensitivity to permit single-particle detection. The minimum concentration at which single-particle detection can be achieved within a given time interval is determined by the particle flux, which is, in turn, dependent upon the maximum achievable flow rate. Two factors are involved. The first is the maximum tolerable pressure across the device; along with the device flow impedance, this sets the highest flow rate that is physically achievable before device failure. The second is the minimum particle residence time that is necessary to achieve a detectable signal above the ambient noise level (the noise "floor"). The former criterion tends to favor devices with larger cross-sectional channel dimensions, while the latter criterion tends to favor smaller, more sensitive, devices.

We consider the minimum requisite residence time for a range of device dimensions, for a specific biological example: Human immunodeficiency virus (HIV) virion detection. HIV is a relatively large virus and, as such, it is one of the more optimally suited viral particles for this technique - it has a diameter of roughly $100 \mathrm{~nm}$ and a mass density of $1.2 \mathrm{~g} / \mathrm{cm}^{3}{ }^{24}$ Suspended microchannel devices actually sense changes in relative density, that is, of the relative increase in mass over that of the fluid displaced by an analyte, referred to here as the mass difference. For an individual HIV virion the mass difference is $\sim 80 \mathrm{ag}$. We will begin by considering SMRs based upon cantilever mass sensors, and will subsequently address doubly-clamped beam devices. We analyze five uniformly scaled cantilever-SMR geometries; their device attributes are summarized in Table I. For each of these five example geometries we perform calculations for two important cases: (a) for an ideal device, for which the dynamic range of motion is determined, at the upper limit, by the onset of mechanical nonlinearity and, at 
the lower limit, by the thermomechanical noise floor and (b) for a more typical device given current technology, for which the practical actuation and transduction efficiencies determine the achievable upper and lower dynamic range limits, respectively. For each of the five geometries, these two cases bound the regimes of performance that is practical at present, and that will be attainable in future with engineering advances. We stress that it is critical to assess practicality in the latter case; what are, ostensibly, "ideal" device parameters can readily verge into the realm of the unattainable. For example, although very stiff devices could, in principle, provide unprecedented sensitivity were they able to be excited to their onset of nonlinearity and measured at the thermomechanical noise floor, in reality they will have miniscule operating amplitudes for feasible excitation amplitudes and, further, will be essentially impossible to transduce down to the level thermomechanical displacement fluctuations. A realistic assessment of such practical limitations provides the essential reality check.

Many methods of actuation and detection are being investigated for microelectromechanical and nanoelectromechanical systems (NEMS/MEMS) devices and a clear winner has not yet emerged. As will be discussed later, many situations may necessitate parallel operation of large device arrays, either to achieve the requisite fluid throughput or to enable simultaneous detection of a multiplicity, or of extremely rare targets. Such large-scale integration is perhaps most easily achieved by employing integrated, "on-chip" electrical actuation and detection of the device resonant motion. To be concrete in our analyses here, we consider electrothermal actuation ${ }^{26}$ and metallic piezoresistive detection, ${ }^{27}$ which have been used to realize the first $200-\mathrm{mm}$-scale "standard NEMS process." ${ }^{28}$ The practical limits considered here include, for displacement transduction, the additional electrical-domain Johnson noise arising from the metallic piezoresistor, and, for actuation, the maximum amplitude attainable with electrothermal actuation set by practical constraints, such as the level of steady-state device heating deemed tolerable. Other popular choices of actuation include electrostatic, which has been used for the majority of SMRs realized to date, ${ }^{7,9,10}$ photothermal actuation, used for the SMR devices of Barton et al., ${ }^{29}$ and piezoelectric which has been implemented successfully for efficient actuation of MEMS (Refs. 30 and 31) and NEMS (Ref. 32) devices but not to date for SMRs. Thus far, detection of SMRs has been performed essentially exclusively optically. More general

discussion of actuation and detection can be found in several MEMS/NEMS review articles. ${ }^{33,34}$ In light of these possibilities, we also consider an intermediate case- $\mathrm{a}$ device limited only by practically attainable piezoresistive detection but assumed to be ideally driven to the onset of nonlinearity (by unspecified means). We include both this case and the fundamental limit (set by thermomechanical motion and the onset of nonlinearity) in all of the figures to provide readers with the means for separately evaluating the consequences of their chosen methods of actuation and detection.

\section{B. Flow-through detection: Frequency stability imposes a fundamental limit to the minimum analyte residence time}

As mentioned previously, flow-through detection requires a minimum particle residence time; this enables sufficient averaging to permit a detectable signal to emerge above the noise floor. This minimum time is determined both by the available dynamic range and by the requisite frequency resolution to detect a threshold mass-density change. Here we perform a detailed analysis of this minimum residence time, focusing on the example provided by Device No. 4 (Table I), chosen for its optimal sensitivity (Fig. 2). The maximum achievable signal is determined either by the onset of nonlinearity or the actuation efficiency. We begin by considering an ideal device, for which actuation to the onset of nonlinearity is achieved. We will first consider silicon-based devices; those fabricated from silicon nitride are considered subsequently. For cantilever-SMRs, the maximum amplitude at the onset of nonlinearity is given by: ${ }^{35}$

$$
\Delta x_{\max }^{\text {cantilever }}=5.46 \frac{\ell}{\sqrt{Q}} \text {. }
$$

For Device No. 4 this yields onset at a displacement of $1.3 \mu \mathrm{m}$. The power spectral density for thermomechanical noise in the displacement domain is given by $S_{x}^{\gamma} \sim 4 k_{B} T Q / \omega K$; for Device No. 4, this translates to $\sqrt{S_{x}^{\gamma}} \sim 1.4 \mathrm{pm} / \sqrt{\mathrm{Hz}}$. The minimum resolvable frequency shift is given by $\delta f / f=1 /[2 Q$ SNR $]$ $\approx \sqrt{S_{x}^{\gamma} / \tau_{a c q}} /\left[2 Q \Delta x_{\text {nonlinearity }}\right]$, , 36 where $\tau_{a c q}$ is the minimum particle residence time and DR is the dynamic range of motion. For flow-through detection, the resolution attained must be sufficient to achieve a detectable mass shift for the individual particle of interest. The requisite fractional shift can be expressed as ${ }^{15,36}$

$$
\delta f / f=-\delta m_{\min } / 2 M_{e f f} .
$$

For HIV, $\delta m_{\min } \sim 80$ ag. Here, $M_{\text {eff }}$ represents the effective mass of the device (which includes its fluidic mass loading); it is ascertained by finite element simulations for the specific device geometries considered (COMSOL). It should be noted that the instantaneous frequency shift is dependent on the position of the target particle along the length channel within the beam. For a particle transiting through the device it is actually the average frequency shift that is relevant for our estimations, given by

$$
\frac{\delta f}{f}=-\frac{\delta m_{\min }}{2 M_{e f f}} \frac{\int_{o_{o}}^{x_{0}+\tau_{a c q} u}\left(Y(x) / Y_{\max }\right)^{2} d x}{\tau_{a c q} u} .
$$

Here, $M_{\text {eff }}$ is the effective mass for point loading at the end of the cantilever, $Y(x)$ is the cantilever deflection at point $x$ along the length, and $u$ is the particle flow velocity. For the threshold case where the minimum required particle residence time is comparable to the actual particle residence time, Eq. (3) can be simplified to the form of Eq. (2), however the effective mass must be interpreted as that for uniform mass loading rather than point loading. Since we are interested in minimum processing times, we will hereafter use this approximation. Within this approximation, Eq. (2) 
can be used with the effective mass replaced by the actual mass of the device plus its entrained fluid, since the weightings of the relative beam positions at position $x$ in Eq. (3) cancel relative to the weightings involved in determining the effective mass. For Device No. 4 the mass of the device plus its incorporated fluid is $\sim 0.10 \mathrm{ng}$. A single HIV virion within the microfluidic channel will thus induce an averaged fractional frequency shift $\delta f / f=385 \mathrm{ppb}$ over the time course of its residence. Note that this is well within the measured $10 \mathrm{ppb}$ frequency stability attained with the remarkable devices of Burg et al. ${ }^{10}$ Combining these expressions gives a minimum particle residence time,

$$
\tau_{a c q}=\frac{S_{x}}{Q^{2} \Delta x_{\max }^{2}} \frac{M_{e f f}^{2}}{\delta m_{\min }^{2}}(\mathrm{SNR})^{2} .
$$

Here $\Delta x_{\max }$ is the maximum achievable amplitude, which is determined by the onset of nonlinearity in the current analysis); $S_{x}$ is the total referred-to-input displacement power spectral density, determined by thermomechanical noise in the current analysis; and SNR is the minimum required signal to noise ratio for detection. In the estimates presented here we impose the conservative requirement that "detectability" assumes the signal should exceed the noise floor by a factor of three, viz., SNR=3. For Device No. 4 this yields a minimum particle residence time of $0.3 \mu$ s for an analyte mass difference $\delta m_{\min }=80 \mathrm{ag}$, and a time of $0.13 \mathrm{~ms}$ for $\delta m_{\min }=4 \mathrm{ag}$. The former condition is chosen to represent the requisite sensitivity for individual HIV virion detection in a pristine environment uncomplicated with other particles, while the latter would enable distinguishing an HIV virion from other constituents in the sample solution provided their mass differences (relative to the displaced water) differ from each other by more than $5 \%$.

For the two smallest devices (geometries Nos. 4 and 5) at 8 ag resolution, the required residence time for data acquisition based on the above analysis is comparable to a single period of oscillation. While it has been established that for frequency-modulated detection in which the cantilever serves as the frequency determining element of an oscillator $^{8-10,37,38}$ the response time is not limited by the device quality factor ${ }^{37}$ as would be the case for conventional amplitude based slope detection, practical limits still remain on the minimum detection time, generally set by the response time of the feedback control loop and the minimum time required to accurately resolve the device frequency. However, it is only for geometries 4 and 5 in the fundamental limit that the minimum acquisition time is sufficiently short for this to be a concern. It will be shown shortly that for these smallest geometries, the maximum throughput is limited not by the measurement response time but by the pressure-limited flow rate through the device, from which a residence time 2000x greater than the previously quoted required acquisition time is determined for geometry No. 4 at 10 psi.

\section{Flow-through detection: Actuation and transduction efficiencies impose a practical limit to minimum analyte residence time}

As mentioned, a practical and currently achievable implementation for integrated actuation and transduction can be realized using thin metal film resistors for both thermoelastic actuation ${ }^{26}$ and piezoresistive detection. ${ }^{27}$ For the latter, we will assume gold film piezoresistors with thickness $50 \mathrm{~nm}$, length $1 \mu \mathrm{m}$, and width $100 \mathrm{~nm}$ are employed. This yields a typical device resistance of 10-100 $\Omega$, and any additional lead resistance is ignored since much thicker films can be employed for the connecting electrodes. It is worth noting that even though the Johnson noise contribution to the total noise power spectral density scales with device resistance, the strain-induced signal itself scales with the device resistance for operation at fixed power. (The fixed-power case is generic since device bias and hence maximum signal are limited by the maximum tolerable heating.) In this limit, the Johnson noise, referred back to the displacement domain, is independent of device resistance to lowest order. The resulting detection sensitivity is much less dependent upon the precise dimensions of the piezoresistor than one might initially expect. A caveat here is that the piezoresistor should be as short as possible to concentrate its overall strain response to within the region of greatest strain at the base of the device. Simultaneously, the length to cross-section ratio should be chosen to allow the strain-sensing resistance to be dominant over lead resistances. These considerations motivate our choice of the piezoresistor geometry employed in this example. We carry out finite element simulations (COMSOL) to estimate the device heating under operational conditions. For geometry No. 4, a bias power of $2 \mathrm{~mW}$ yields a maximum temperature rise of $9{ }^{\circ} \mathrm{C}$ at the fluid-silicon interface, which is deemed acceptable. (For other geometries tolerable bias levels range from $0.6 \mathrm{~mW}$ for the smallest device to $11 \mathrm{~mW}$ for the largest.) The maximum temperature rise in the fluid will be somewhat less than this due to incomplete thermalization of the fast-flowing fluid, and from the fluid-mediated device cooling it provides. At this $2 \mathrm{~mW}$ bias, the Johnson noise contribution to the total noise voltage power spectral density is $\sqrt{S_{V}^{J}} \sim 1 \mathrm{nV} / \sqrt{\mathrm{Hz}}$. Referred back to the displace-

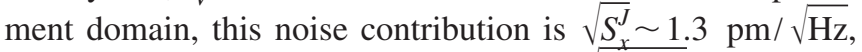
for a combined noise spectral density, $\sqrt{S_{x}^{J}+S_{x}^{\gamma}}$ only about $40 \%$ greater than the fundamental limit set by thermomechanical fluctuations. The induced strain in the piezoresistive region from thermoelastic actuation is given by

$$
\left\langle\varepsilon_{x x_{\mathrm{PR}}}\right\rangle=Q \alpha \Delta T \frac{E_{\text {actuator }}}{E_{\text {device }}} \frac{\left\langle\varepsilon_{x x}^{*}\right\rangle_{\mathrm{PR}}\left\langle\varepsilon_{x x}^{*}+\varepsilon_{y y}^{*}+\varepsilon_{z z}^{*}\right\rangle_{\text {actuator }}}{\left\langle\varepsilon^{i j^{*}} \varepsilon_{i j}^{*}\right\rangle_{\text {device }}}\left(\frac{V_{\text {actuator }}}{V_{\text {device }}}\right) .
$$

Here $\left\langle\varepsilon_{x x}\right\rangle_{\mathrm{PR}}$ is the average induced strain in the piezoresistive region (in the direction of current flow), $\left\langle\varepsilon_{x x}^{*}\right\rangle_{\mathrm{PR}}$ is the average strain in the piezoresistive region (in the direction of current flow) for a normalized amplitude of oscillation, $\left\langle\varepsilon_{x x}^{*}\right.$ $\left.+\varepsilon_{y y}^{*}+\varepsilon_{z z}^{*}\right\rangle_{\text {actuator }}$ is the average total strain in the actuator for the same normalized amplitude of oscillation, and $\left\langle\varepsilon^{i j} \varepsilon_{i j}^{*}\right\rangle_{\text {device }}$ is the average product of strain tensors over the 


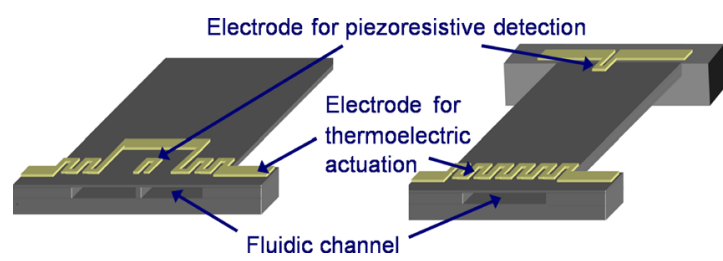

FIG. 1. (Color online) Schematic of a silicon SMR with integrated thermoelectric actuation and piezoresistive detection. Left: cantilever, right: doubly-clamped beam.

entire device for the same normalized amplitude of oscillation. As usual, $Q$ represents the device quality factor; $\alpha$ is the thermal coefficient of linear expansion for the metallic (gold) actuator; $\Delta T$ the temperature rise within the metallic actuator, which has been shown by numerical simulations to be roughly uniform for excitation frequencies below the inverse thermal time constant of the drive loop; ${ }^{39} E_{\text {actuator }}$ and $E_{\text {device }}$ are the Young's moduli for the actuator and device, respectively; and $V_{\text {actuator }}$ and $V_{\text {device }}$ are the respective volumes. ${ }^{26}$ As the actuation efficiency is strongly dependent on the actuator's volume relative to the total device volume, we assume a somewhat thicker $(80 \mathrm{~nm})$ gold film is used compared to that of the piezoresistor. This increased thickness also provides efficient cooling, allowing the device to operate at higher frequencies. Given that Au has greater thermal conductivity than $\mathrm{Si}$, much of the cooling for this geometry will occur through the metal film. The optimum actuation length is determined by a number of factors. If the actuator is too short then the actuation is inefficient due to the $V_{\text {actuator }} / V_{\text {device }}$ term. However, if it is too long a reduction in $\left\langle\varepsilon_{x x}^{*}+\varepsilon_{y y}^{*}+\varepsilon_{z z}^{*}\right\rangle_{\text {actuator }} V_{\text {actuator }}$ results, since terms under both compressive and tensile stress cancel. This is particularly significant for higher order modes. Before this begins to play a role, a decrease in signal will be observed with increasing actuator length due to decreased cooling efficiency. The frequency of thermal roll-off in actuation efficiency scales as roughly the square of the inverse of the actuator length. For $80 \mathrm{~nm}$ thick electrodes, a $0.25 \mu \mathrm{m}$ long gold actuator has thermal roll-off at roughly $40 \mathrm{MHz} .^{26}$ In practice, for fundamental mode operation, the actuation efficiency is optimized when the thermal roll-off is roughly equal to the operating frequency. We employ this fact as a design constraint here. Since the devices of interest here are much wider than those of Bargatin et al.,${ }^{26}$ we assume a meandering layout is employed; this maximizes surface coverage of the actuator while maintaining reasonable resistance values (Fig. 1). For the cantilever-SMR geometry of Device No. 4 (actuator length: $1.7 \mu \mathrm{m}$, actuator width, $0.27 \mu \mathrm{m}$, with one loop on either side of the piezoresistor), a $9{ }^{\circ} \mathrm{C}$ maximum temperature rise in the gold regions (from which the flowing fluid is partially thermally isolated by the silicon) thermoelastic actuation should provide a maximum deflection of $\sim 300 \mathrm{~nm}$. This corresponds to an average strain in the piezoresistive region of $7.5 \times 10^{-4}$, and yields displacements roughly a factor of ten below the onset of nonlinearity. For Device No. 4, if we incoherently sum the contributions of both thermomechanical and Johnson noise, and assume the device is operated it at the maximum deflection achievable with thermoelastic actuation, a minimum particle residence time of 5 ms is required to achieve 4 ag resolution. This can be compared with a much shorter time of $0.13 \mathrm{~ms}$ obtained were one to assume an "ideal" device is realizable. This significant difference serves to illustrate the importance of careful assessment of practical limitations.

\section{Flow-through detection: Circumventing long measurement times may require analyte preconcentration or parallel processing}

Two considerations enter into the determination of the maximum flow rate. These are the minimum particle residence time required to achieve a detectable signal, as discussed above, and the maximum tolerable pressure drop across the device. Continuing with the previous example, a residence time of $0.13 \mathrm{~ms}$ corresponds to a maximum volumetric flow rate of $6 \mathrm{nl} / \mathrm{min}$ through a single device, whereas $0.16 \mathrm{nl} / \mathrm{min}$ is obtained for the previously calculated practical limit of $5 \mathrm{~ms}$. If we assume fluid flow through the device can be modeled as simple Hagen-Poiseuille flow, ${ }^{40}$ achieving a flow rate of $6 \mathrm{nl} / \mathrm{min}$ will require a pressure drop of $\sim 28 \mathrm{psi}$ across the device. This is at the upper limit for realistic pressures attainable with existing device technologies. If we more conservatively estimate that the maximum tolerable pressure is $\sim 10 \mathrm{psi}$, then the maximum achievable flow rate is $\sim 1 \mathrm{nl} / \mathrm{min}$. In Fig. 2, we plot the maximum flow rate, determined by considering both the maximum tolerable pressure and the minimum required residency time. Devices of similar geometry are routinely operated at pressures of order 10 psi (Ref. 18) shown as a solid line in Fig. 2. For 80 ag resolution at $10 \mathrm{psi}$, we see that the maximum achievable flow rate is pressure limited for both the fundamental and practical limits. A dotted line is used to indicate the limit of detection (LOD) which could be achieved at 1000 psi. For flow-through detection, target particles can be distinguished from solution constituents only to within the accuracy of the mass measurement. For example, 80 ag resolution might allow a single virion to be detected but would not allow it to be distinguished from a 100 ag particle in the solution. Accordingly, in Fig. 2 we also plot the maximum flow rate for detection in a "pristine" solution unpopulated by competing analytes (requiring only 80 ag resolution), and that required to permit distinguishing particles whose mass difference (relative to the water displaced) differs from the target by more than 5\% (requiring a more stringent 4 ag resolution). For cases where discrimination between particles that are extremely close in mass is essential, it may not be possible to attain sufficient mass resolution with any realizable device geometry, and a separation-based method may become necessary. For such cases, rather than employing flow-through detection, discrimination could be achieved by selective prefiltering of the sample solution, or by affinity-based capture at the sensor itself. The mutually competing limitations-of flow rate, stemming from device geometry; and of the requisite minimum residence time $\tau_{a c q}$, enabling single-particle detection-yield an optimum geometry that is roughly of dimensions represented by Device No. 4 .

In our preceding analysis we have not included the effects of long-term drift. We also neglect possible contributions from $1 / f$ noise, having assumed the operating fre- 
80 ag resolution

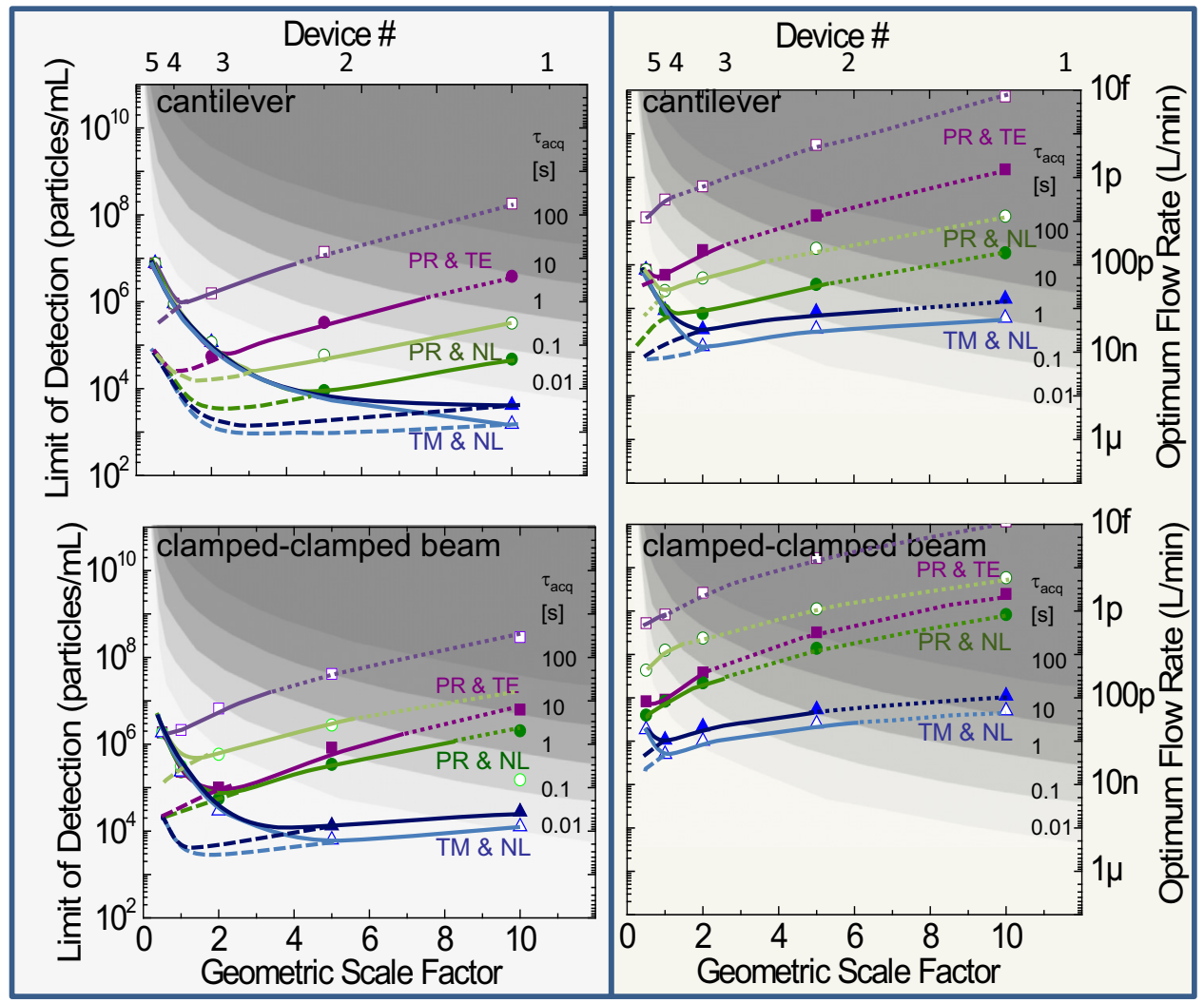

FIG. 2. (Color online) LOD for flow-through detection when determined by device sensitivity (minimum residence time required for single-particle detection) and device geometry. The optimum flow rate (right axis) at which target can be introduced to the device is determined by both the device geometry (dominant for small devices) and $\tau_{a c q}$, the minimum residence time required for the device to achieve single-particle detection (dominant for large devices). The minimum residence times are indicated by the gray shading in each plot. Values for the geometric scale factor assume uniform scaling relative to Device No. 4 (Table I). To compute the LOD (left axis) it is assumed that ten particles must be detected in 10 min. Left: 80 ag resolution (would allow HIV virion detection in a pristine environment). Right: 4 ag resolution (would allow HIV virions to be distinguished from other particles in solution provided the mass difference was $>5 \%$ ). Top: cantilevers, Bottom: doubly- clamped beams. $\mathbf{\Delta}$ (blue triangle) TM and NL: LOD, fundamental limit (signal to noise ratio limited by the onset of nonlinearity and thermomechanical noise). (green circle) PR and NL: LOD for a device with integrated piezoresistive read-out (maximum drive set by onset of nonlinearity). (purple square) PR and TE: LOD, practical limit (piezoresistive read-out and thermoelastic actuation). Solid symbols ( $\boldsymbol{\Lambda}$, - $\mathbf{\square})$ : silicon devices. Hollow symbols $(\triangle, \bigcirc, \square)$ : silicon nitride devices. The solid lines are a guide to the eye and correspond to a maximum operating pressure of 10 psi. Dashed lines (no symbols) are used to indicate the LOD if a maximum operating pressure of 1000 psi is permitted. Dotted lines are used to highlight regimes where particle residence times $\left(\tau_{a c q}\right)$ exceed $1 \mathrm{~s}$ and long-term drift (not included in the model) becomes an increasing concern. Several conclusions can be taken from the plots. First, there is an optimal geometry that balances the higher sensitivity of the small devices against the higher volumetric flow rate achievable with the large devices. Second, the material differences between silicon and silicon nitride do not substantially affect device performance, except for the practical limit where the reduced thermal conductivity and increased stiffness of the nitride degrades the actuation and detection efficiency. We stress that the processing of a $1 \mu \mathrm{l}$ sample volume (required to detect ten particles at a concentration of $10^{4} \mathrm{particles} / \mathrm{ml}$ through a single device at $170 \mathrm{pl} / \mathrm{min}$ (optimal flow rate for achieving 4 ag resolution) would require four days. Achieving practical analysis times will require alternative approaches, such as preconcentration and use of large device arrays.

quency is chosen sufficiently high as to circumvent such contributions. These considerations could present a challenge if integration times greater than $1 \mathrm{~s}$ are envisaged. Except for the largest devices, all the SMRs considered here have sufficient sensitivity to achieve individual HIV virion detection (80 ag resolution) with particle residence times shorter than $\sim 1$ s. However, only the smallest devices can reach 4 ag resolution within a $\sim 1 \mathrm{~s}$ averaging time.

If we assume acquisition of ten detection events is the threshold permitting a statistically meaningful measurements, then the optimal flow rate can be directly related to the LOD within a convenient, fixed measurement time. We set this time to be $10 \mathrm{~min}$; the implications of this are displayed in Fig. 2. For Device No. 4, a LOD of 9 $\times 10^{5}$ particles $/ \mathrm{ml}\left(1.5 \times 10^{4}\right.$ particles $/ \mathrm{ml}$ if the $10 \mathrm{psi}$ pressure limit is not included) is obtained for detection in "pristine" solution (80 ag resolution), whereas an LOD of $6 \times 10^{6}$ particles $/ \mathrm{ml}$ is obtained for detection providing the ability to distinguish particles whose mass difference (relative to displaced water) differs by more than 5\% (4 ag resolution). These considerations can be recast as the requisite measurement time to evaluate a sample with a specific target concentration. For example, to detect ten particles from a sample with a concentration of $10^{4}$ particles $/ \mathrm{ml}$ without preconcentration, one finds that $1 \mu \mathrm{l}$ of sample must be processed through the device. Here we make the optimistic assumption that every particle flowing through the device is detected. For Device No. 4, the maximum practical flow rate to achieving 4 ag resolution is, from Fig. 2, approximately $170 \mathrm{pl} / \mathrm{min}$. Processing $1 \mu \mathrm{l}$ through a single device at this flow rate would require approximately four days. This illustrates that for flow-through detection to be useful at these 
concentrations, parallel processing (that is, arrays of SMRs) or preconcentration will be required. For example, with both thousand-fold preconcentration and parallel operation of an array of 50 devices the same sample volume could be processed in about $6 \mathrm{~s}$. We also anticipate that there is significant possibility for further optimization of the actuation and detection efficiencies with piezoelectric actuation and detection. $^{41,42}$ The optimum volumetric flow rate for a device operating at the fundamental limit (with 4 ag resolution) is $7.4 \mathrm{nl} / \mathrm{min}$ (achieved for the silicon nitride cantilevers in geometry No. 3). At this sensitivity an array of 50 devices with thousand-fold preconcentration could process the requisite sample volume in $\sim 0.2 \mathrm{~s}$. However, even with such advances, processing of the requisite sample volume by a single device without aid of preconcentration would still remain impractically slow, of order $2 \mathrm{~h}$.

\section{E. Flow-through detection: Selection of device materials can affect device sensitivity}

The materials used to fabricate an SMR device affect its performance. For the smallest devices, the LOD is determined by the maximum flow rate, which is, in turn, set by the maximum tolerable pressure that can be applied. This is not particularly dependent on the material properties (Fig. 2). For larger devices, however, the maximum flow rate is determined by the sensitivity - which is indeed dependent upon material properties. For "ideal" devices with performance set by fundamental limits, a slight improvement is seen for silicon nitride devices, primarily due to the higher Young's modulus compared to silicon and the corresponding increase in resonance frequency. However, we find this improvement is modest, typically only of order two. By contrast, for devices limited by practical considerations, silicon nitride yields inferior performance. This stems from the decreased actuation and transduction efficiencies, which are a consequence of the material's reduced thermal conductivity-for geometry No. 4 COMSOL simulations indicate a temperature rise of $9{ }^{\circ} \mathrm{C}$ at the silicon/water interface for a bias across the piezoresistor of $220 \mu \mathrm{W}$ as compared with $2 \mathrm{~mW}$ for a silicon device-and increased stiffness and density compared to that of $\mathrm{Si}$.

\section{F. Flow-through detection: Comparison of cantilevers and doubly-clamped beams in the fundamental limit, for flow-through detection applications}

The discussion so far has focused on cantilever-based SMR devices. With several additional considerations, most of the calculations presented above also relate to doublyclamped beams. In our discussion of fundamental limits, we stated that the maximum deflection is generally set by the onset of nonlinearity. For a doubly-clamped beam SMR, the deflection at the onset of nonlinearity is given by ${ }^{43}$ $\Delta x_{\text {nonlinearity }}=\sqrt{2} / \sqrt{\alpha Q \sqrt{3}}$. Here, $\Delta x_{\text {nonlinearity }}$ is the deflection of the mid-point of the beam at the $1 \mathrm{~dB}$ compression point (the point at which the signal is $1 \mathrm{~dB}$ lower than expected for the case of a purely linear response), $t$ is the device thickness, $\alpha=0.05 A / I, A$ is the cross-sectional area, and $I$ is the moment of inertia. For Device No. 4, this indicates that
$\Delta x_{\text {nonlinearity }}=27 \mathrm{~nm}$; this value should be compared with the similar onset occurring at a much larger deflection, $\sim 1.3 \mu \mathrm{m} \mathrm{rms}$, for the more compliant cantilever geometry. The thermomechanical displacement noise spectral density, $S_{x}^{\gamma}$, is roughly a factor of 36 smaller for the doubly-clamped beam than for the cantilever due to its higher frequency and stiffness. The expression for the minimum residence time [Eq. (4)], shows that for devices of equal quality factor, resonator mass, and minimum detectable mass, the relative particle residence times are given by $\tau_{a c q}^{\text {cantilever }} / \tau_{a c q}^{\text {beam }}$ $=\left(S_{x}^{\text {cantilever }} / S_{x}^{\text {beam }}\right)\left(\Delta x_{\max }^{\text {beam }} / \Delta x_{\max }^{\text {cantilever }}\right)^{2} \sim 0.15$. This implies that the maximum tolerable volumetric flow rate, limited by the requisite averaging time (set by the device mass sensitivity), is about sevenfold larger for a cantilever than for a doubly clamped beam. This is a direct consequence of fact that the greater dynamic range of motion for cantilevers makes their frequency-fluctuation noise lower, compared with that of doubly-clamped beams of similar dimensions.

\section{G. Flow-through detection: Comparison of cantilevers and doubly-clamped beams in the practical limit, for flow-through detection applications}

Comparison between practical cantilever and doublyclamped beam devices is straightforward if one evaluates the strain induced at the onset of nonlinearity. For this analysis we assume metallic piezoresistive read-out is employed, and that the resonators are driven to the onset of nonlinearity. For a doubly-clamped beam with geometry of Device No. 4, its $27 \mathrm{~nm}$ rms deflection at the onset of nonlinearity corresponds to an average strain in the piezoresistive region of $\sim 5$ $\times 10^{-4}$. A cantilever device with the same geometry would develop, at its much larger $1.3 \mu \mathrm{m}$ (rms) onset of nonlinearity, an average strain of $\sim 3 \times 10^{-3}$. The Johnson noise from the piezoresistors will be identical for both devices, so the relative particle residence times can be compared by the expression $\tau_{a c q}^{\text {cantilever }} / \tau_{a c q}^{\text {beam }}=\left[\varepsilon_{\max }^{\text {beam }} / \varepsilon_{\max }^{\text {cantilever }}\right]^{2}$, which is of order 0.02 for the present example. Within the sensitivity-limited regime, this corresponds to a 50-fold improvement in the LOD for the cantilever-based SMR, as compared to that of a doubly-clamped beam device.

Employing current technology, a practical device might be constructed using piezoresistive detection and thermoelastic actuation. The maximum achievable strain via thermoelastic actuation is similar for both cantilevers and doubly-clamped beams. Our finite element simulations of devices with the dimensions of Device No. 4 indicate a maximum strain of $4.4 \times 10^{-4}$ is attained for the doubly-clamped beam and $7.5 \times 10^{-4}$ for the cantilever. For piezoresistive readout, assuming the device is Johnson noise limited, the relative particle residence time is given by $\tau_{a c q}^{\text {cantilever }} / \tau_{a c q}^{\text {beam }}$ $=\left(\varepsilon_{\max }^{\text {beam }} / \varepsilon_{\max }^{\text {cantilever }}\right)^{2} \sim 0.34$. This indicates that the maximum volumetric flow rate determined by device sensitivity is roughly threefold larger for a cantilever than for a doublyclamped beam. When both Johnson noise and thermomechanical noise are considered, along with thermoelastic actuation, then the cantilever shows only a twofold improvement in LOD over the doubly-clamped beam. In fact, for the cantilever geometry in the practical limit at 80 ag resolution and a maximum operating pressure of $10 \mathrm{psi}$, 
the flow rate of Device No. 4 is actually pressure-limited and, as such, the doubly-clamped beam is preferable. Threefold greater maximum flow rates can be obtained with the doubly-clamped beam SMR than with the cantilever device due to the flow channel architecture. For the former this can be half the length and twice the width as for a comparable cantilever device, since the microchannel in a doublyclamped device need not loop back on itself. Doublyclamped beam SMRs have two additional apparent advantages: namely, that their design is more robust against fabrication- and shock-induced stiction, and they are less likely to clog under conditions of operation than the cantilever-SMRs that have a U-shaped flow channel. The maximum achievable flow rates for cantilever and doublyclamped beam geometries are both shown in Fig. 2. At 10 psi, for the larger, sensitivity-limited, devices [Devices Nos. 1,2 , and 3], the cantilever outperforms the doubly-clamped beam, whereas for the smallest, pressure-limited devices [Devices Nos. 4 and 5], the doubly-clamped beam yields the best performance. At 1000 psi and 80 ag resolution, the cantilever outperforms the doubly-clamped beam for all but the smallest device. For 4 ag resolution sensitivity considerations become correspondingly more important, favoring cantilever devices for all five size scales even at 10 psi.

\section{ANALYSIS OF DETECTION USING AFFINITY-CAPTURE: INDIVIDUAL VIRIONS}

For affinity-capture detection, target particles are captured on a biofunctionalized surface of the device. In contrast to flow-through operation, the flow rate and resulting analysis time for affinity-capture operation is not determined by the minimum particle residence time required for singleparticle detection. Instead, the sensitivity is determined by the flow rate, in turn determined by geometry, maximum tolerable pressure, and by the kinetics of diffusion and binding.

With affinity capture, the time required for signalaveraged detection of immobilized particles is not generally a significant consideration. Accordingly, understanding the kinetics of the affinity-capture process is critical; it can play an equally significant role as the device mass sensitivity in determining the SMR's concentration sensitivity. Maximal capture of target in a fixed time interval is achieved with high flow rates, which allow convection to dominate over diffusion. ${ }^{11-13,34,44}$ In this regime the boundary layer, across which analytes must diffuse to bind to the surface, is minimized. The maximum, pressure-limited, flow rates that can be achieved in the SMR devices considered here are of order $0.3 \mathrm{nl} / \mathrm{min}$ to $2 \mu \mathrm{l} / \mathrm{min}$. Since we are interested in fast detection $(<10 \mathrm{~min})$, it is reasonable to consider single pass detection within the device volume. Throughout this analysis, our assumption will be that microliters of sample are at our disposal, hence we assume fluid will not need to be recirculated to maximize the likelihood of analyte capture. Hence, we need only consider the possibility of analyte depletion near the functionalized capture surface, and not within the bulk of the sample. We follow the approach presented by Squires and Manalis. ${ }^{11}$ Following their analysis, it can be verified that convection dominates over diffusion by considering two Peclet numbers. The first, $\mathrm{Pe}$ channel $=t_{D} / t_{C}$ $\sim Q_{V} /\left(D w_{c}\right)$ (Ref. 11) ranges from $5 \times 10^{2}$ to $8 \times 10^{5}$ for the flow rates considered here arising from an assumed 5 psi pressure drop across the device. In this expression, $t_{D}$ and $t_{C}$ are the diffusion and convection times with respect to the channel thickness, $d_{c}{ }^{45}$ Also, $Q_{V}$ is the volumetric flow rate, $D$ is the diffusion constant (a value of $2.2 \times 10^{-12} \mathrm{~m}^{2} / \mathrm{s}$ is assumed for HIV virions ${ }^{46}$ ), and $w_{c}$ is the channel width. The second Peclet number, $\mathrm{Pe}_{\substack{\text { sensor } \\ \text { depth }}}=t_{D} / t_{C} \sim 6\left(L / d_{c}\right)^{2} \mathrm{Pe}$ channel, compares diffusion to convection times relative to the "sensor depth," L. By sensor depth we mean the length of the (functionalized) sensor capture region along the direction of fluid flow. For SMRs, $L$ will usually be the entire microchannel path length within the sensor. Note that it is important to functionalize only the path within the vibratory device, so as to circumvent analyte depletion from the adjacent (anchored) regions that are not sensed.

For the five device geometries analyzed here, the sensor depth is large compared to the channel thickness, that is $L d_{c}$, hence $\mathrm{Pe}_{\text {sensor }} \gg 1$. In this regime, where $\mathrm{Pe}_{\text {channel }}^{\text {thickness }}$ and $\mathrm{Pe}_{\text {sensor }}^{\text {depth }}$ are both much greater than one, the number of particles reaching the sensor per unit time is given by

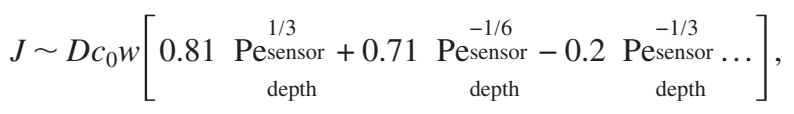

where $c_{0}$ is the concentration particles in solution. ${ }^{11,47,48}$ The fraction of the analyte flux that reaches the functionalized capture area is therefore given by $f=J /\left(Q_{V} c_{o}\right)$. This ranges from $0.2 \%$ for the largest devices to $10 \%$ for the smallest devices. We further assume that $10 \%$ of these particles reaching the surface actually bind to the device and, further, that a $1 \mathrm{~Hz}$ bandwidth is used. Under these conditions, when both Johnson noise and thermoelastic actuation are considered, single-particle detection appears possible for all but the largest device. For this largest of devices the LOD is, at best, the signal that would arising from the binding of three particles.

To acquire a statistically meaningful measure of concentration, we impose the criterion that a minimum of ten particles must be detected. The resulting minimum concentration sensitivities are shown in Fig. 3. The improvement in concentration sensitivity for the larger devices arises primarily from their greater channel width (and hence surface area for capture), allowing more particles to be captured within the same time interval. This increase is observed solely because HIV virions are sufficiently massive that, even with the largest device, single-to few-particle sensitivity can be attained. However, for smaller viruses such as HCV, Polio, and Parvo, there is an improvement in performance for the smaller devices arising from their enhanced sensitivity. In sensing these lighter targets with the smallest devices, however, considerations of particle flux and flow rate can, once again, play a limiting role. 


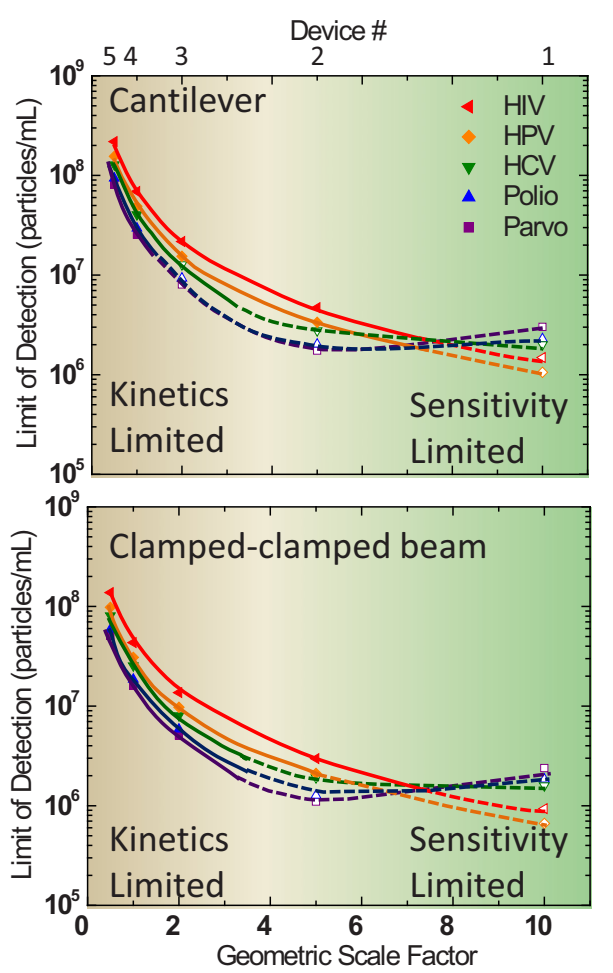

FIG. 3. (Color online) Concentration sensitivity for virion detection with capture. A threshold of ten captured particles in $10 \mathrm{~min}$ is assumed (except where greater numbers of particles are required to reach the sensitivity threshold). Solid lines are used for single-particle sensitivity, dashed lines if the device sensitivity is insufficient for single-particle detection. Only the practical limit is shown here. For practical analyses, preconcentration would enable attainment of typical desired target thresholds of order $\sim 10^{4} / \mathrm{ml}$.

\section{ANALYSIS OF DETECTION USING AFFINITY- CAPTURE: PROTEINS}

A similar analysis may be used to estimate the sensitivity of SMR devices employed for protein detection. Since these devices generally do not have the sensitivity to detect individual proteins, affinity-based capture becomes necessary. To be concrete, we take prostrate specific antigen (PSA) for our prototypical protein. PSA is a $34 \mathrm{kDa}$ protein, ${ }^{49}$ with a partial specific volume of $0.744,{ }^{50}$ for a mass difference (relative to displaced water) of $8.7 \mathrm{kDa}$. The LOD, here couched as the minimum number of proteins that can be detected, are plotted for our five device geometries in Fig. 4. Sensitivity down to the level of tens of proteins is possible for the smallest devices, even in the practical limit. As is evident from Fig. 4, SMR cantilever devices performing at the fundamental limit should achieve the potential of single-protein sensitivity.

As in our previous considerations, the ultimate concentration sensitivity will be determined by both the mass sensitivity of the device and the kinetics of analyte capture. For the latter, analyte diffusion and reaction kinetics both play a role. However, in almost all practical situations of relevance, we will show that SMR devices operate in the reactionlimited regime. To evaluate this, we estimate protein diffusion in the same manner as for the HIV detection analysis described previously, assuming a diffusion coefficient $D$ $=8.5 \times 10^{-11} \mathrm{~m}^{2} / \mathrm{s} .{ }^{49}$ The Peclet numbers still remain significantly greater than one, although they are less than for HIV detection-Pe channel ranges from 26 for the smallest de-
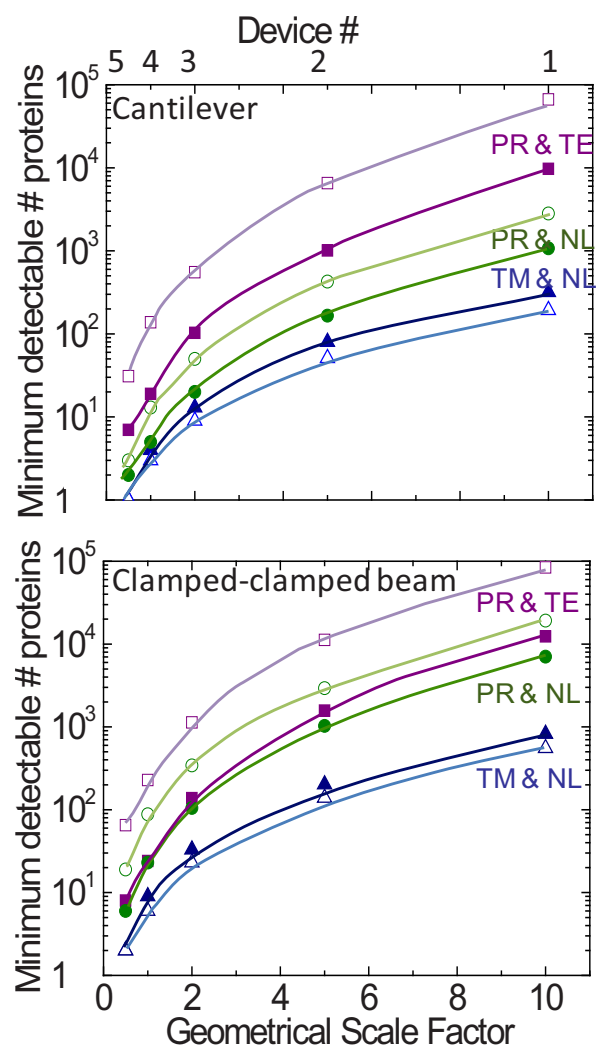

FIG. 4. (Color online) Sensitivity limit for protein detection with capture. A protein mass of $34 \mathrm{kDa}$ is assumed (corresponding to PSA); $\boldsymbol{\Delta}$ (blue triangle) TM and NL: Minimum detectable number of proteins, fundamental limit (signal to noise ratio limited by the onset of nonlinearity and thermomechanical noise) (green circle) PR and NL: minimum detectable number of proteins for a device with integrated piezoresistive read-out (maximum drive set by onset of nonlinearity). $\square$ (purple square) PR and TE: minimum detectable number of proteins, practical limit (piezoresistive read-out and thermoelastic actuation). The device dimensions are uniformly scaled relative to that of Device No. 4 (Table I). Solid symbols $(\boldsymbol{\Delta}, \mathbf{O}, \mathbf{\square})$ : silicon device. Hollow symbols $(\triangle, \bigcirc, \square)$ : silicon nitride device. The solid lines are a guide to the eye.

vices to $2 \times 10^{4}$ for the largest of devices. To understand the binding kinetics, it is important to consider another dimensionless parameter, the Damkohler number, ${ }^{11}$

$$
D a=\frac{k_{o n}}{f} \frac{b_{m}}{Q_{V}} \frac{10^{-3} \mathrm{~m}^{3} / 1}{N_{A}},
$$

where $N_{A}$ is Avogadro s number, and $k_{o n}$ is the on rate, for which $k_{\text {on }}=4.1 \times 10^{4} \mathrm{M}^{-1} \mathrm{~s}^{-1}$ is used, corresponding to PSA binding to anti-PSA monoclonal antibody. ${ }^{51}$ its monoclonal antibody assumed to have a value of $1.2 \times 10 \mathrm{M}^{-1} \mathrm{~s}^{-1}$. This corresponds to the measured rate constant for human T-cell receptor CD4 binding to anti-CD4 monoclonal antibody. ${ }^{52}$ Also, $b_{m}$ is the total number of binding sites. For each of the five geometries, we estimate $b_{m}=\beta A$, assuming a typical receptor surface density of order $\beta=2 \times 10^{4} / \mu \mathrm{m}^{2}$, ${ }^{11,53}$ with $A$ representing the active (functionalized) surface area within the device microchannel. This produces a value for the Damkohler number ranging from $3 \times 10^{-3}$ for the smallest device, to $1 \times 10^{-2}$ for the largest. The concentration near the sensor is given by $c_{s}=c_{0} /(1+D a)$ where $c_{0}$ is the bulk (undepleted) concentration. For the geometries considered, this gives $c_{s} \geq 0.9 c_{0}$ for all of the devices considered here. In 


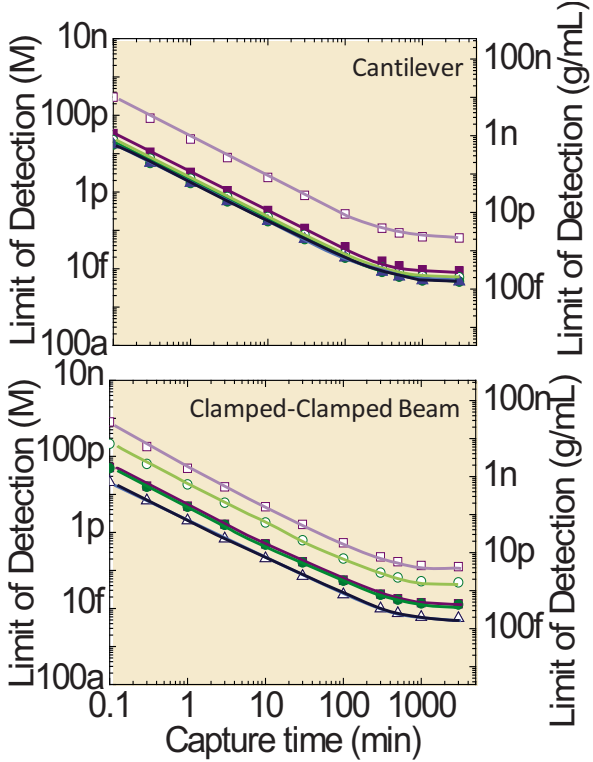

FIG. 5. (Color online) LOD for protein capture using SMRs; illustrating the intrinsic time dependence of concentration sensitivity. The time-dependent LOD for PSA detection with device geometry No. 4 is shown. LOD improves with time until equilibrium saturation is reached, an interval of order $2 \mathrm{~h}$. $\boldsymbol{\Delta}$ (blue triangle) TM and NL: LOD, fundamental limit (signal to noise ratio limited by the onset of nonlinearity and thermomechanical noise) (green circle) PR and NL: LOD for a device with integrated piezoresistive read-out (maximum drive set by onset of nonlinearity). (purle square) PR and TE: LOD, practical limit (piezoresistive read-out and thermoelastic actuation). Solid symbols $(\boldsymbol{\Lambda}, \boldsymbol{\bullet}, \boldsymbol{\square})$ : silicon device. Hollow symbols $(\triangle, \bigcirc$, $\square)$ : silicon nitride device.

other words, analyte depletion is minimal in this reactionlimited regime. From this we can estimate the number of bound molecules at time $t$, as

$$
\frac{b(t)}{b_{m}}=\frac{c_{s} K_{C}}{1+c_{s} K_{C}}\left[1-\exp \left(k_{o n} c_{0}+k_{o f f}\right)\right] .
$$

Here $k_{\text {off }}$ is the disassociation rate constant, for PSA $k_{\text {off }}$ $=4.5 \times 10^{-5} / \mathrm{s}$ (Ref. 51) and $K_{C}=k_{\text {on }} / k_{\text {off }}$ is the equilibrium constant. Since the minimum required number of bound proteins $b(t)$ is determined by the sensitivity of the device (Fig. 4), Eq. (8), allows us to estimate the LOD for our example device geometries. These are actually time-dependent, as illustrated in Fig. 5 for Device No. 4. For operation at short time scales, $<10 \mathrm{~min}$, measurements remain in the nonequilibrium, mass-transport-limited regime. Nevertheless, exceptional sensitivity at the picomolar scale is attained. Figure 6 shows the concentration sensitivity of all five devices for a 10 min capture time. Again, there is a competition evident between pressure-limited flow rate and the device sensitivity; optimum performance is predicted for Device No. 4 in the practical limit, which attains a projected sensitivity of 1.5 $\mathrm{pM}(0.23 \mathrm{ng} / \mathrm{ml})$. This value is attained for a single device without preconcentration. This is very promising performance when compared against other current state-of-the-art technologies. ${ }^{34}$

\section{CONCLUSIONS}

Our analysis shows that NEMS-based and MEMS-based SMR devices can provide extremely high sensitivity, suffi-

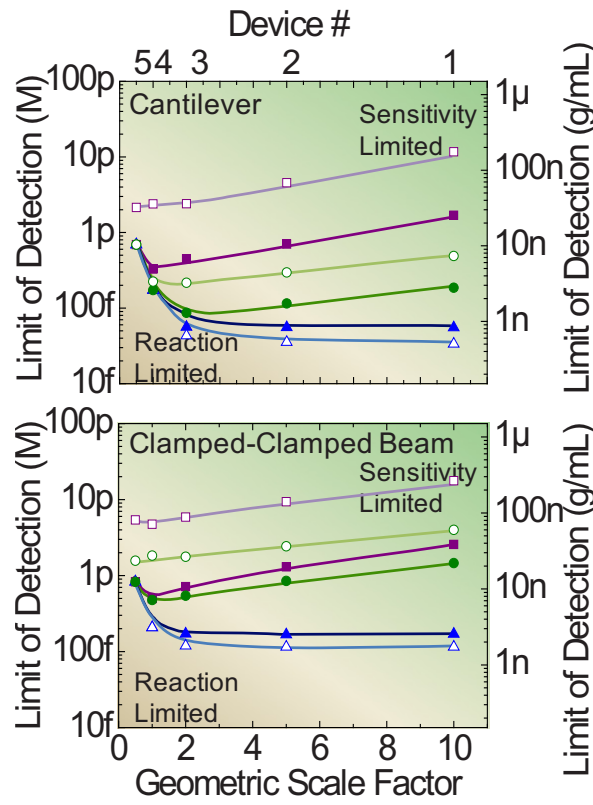

FIG. 6. (Color online) LOD for protein capture using SMRs. Concentration sensitivity for a 10 min capture time for the devices of Table I (top: cantilevers, bottom: doubly-clamped beams). For the practical devices (purple) an improvement in concentration sensitivity is seen as the dimensions are reduced for all but the smallest device. Reducing the device dimensions below this threshold is counterproductive as device performance degrades due to the reduced capture cross-section. $\boldsymbol{\Delta}$ (blue triangle) TM and NL: LOD, fundamental limit (signal to noise ratio limited by the onset of nonlinearity and thermomechanical noise) - (green circle) PR and NL: LOD for a device with integrated piezoresistive read-out (maximum drive set by onset of nonlinearity). (purple square) PR and TE: LOD, practical limit (piezoresistive read-out and thermoelastic actuation). Solid symbols $(\boldsymbol{\Lambda}, \boldsymbol{\bullet}$,

口): silicon device. Hollow symbols $(\triangle, \bigcirc, \square)$ : silicon nitride device.

cient to access new and interesting regimes for biodetection. The results presented here demonstrate that even with the largest, MEMS-scale devices of Table I, such as Device No. 2 with length $=100 \mu \mathrm{m}$ and $t=3.5 \mu \mathrm{m}$, it is feasible to achieve single-particle detection for a large virus such as HIV. For smaller molecular-scale analytes such as proteins, detection of less than 1000 (accumulated) particles appears possible. Much smaller devices such as Device No. 4, with length $=20 \mu \mathrm{m}$ and $t=700 \mathrm{~nm}$, have the potential to achieve even higher sensitivity, down to the few-to-single protein regime.

We have shown that in the flow-through mode of SMR operation, the sensors themselves can be designed to have exquisite mass sensitivity, sufficient for the detection of individual analytes. In the final analysis, however, the dominant limitation to attainable performance is the minute volume of sample fluid that a single device can process per unit time. Achieving clinically useful analyte concentration sensitivities on practical time scales proves difficult without both preconcentration and significant device parallelization.

In optimizing affinity-capture based SMRs there are two competing considerations: larger devices can process more sample volume per unit time, so the flux to the sensor region can be optimized; whereas smaller devices provide higher mass sensitivity. Optimization of performance must simultaneously counterbalance these two considerations. Also, affinity-capture based SMRs are essentially always in the 
reaction-limited regime. Hence, in direct contrast to the flow-through mode of operation, affinity-capture SMR devices are not generally limited by the analyte flux. For this case then, it is maximizing capture cross-section that is paramount.

Despite these issues, we have demonstrated that SMRs have the potential to achieve protein concentration sensitivity of order picomolar in less than $20 \mathrm{~min}$. This compares favorably to the detection sensitivities of most commercial methods (e.g., Enzyme-linked immunosorbent assay), which are of order of picomolar but suffer from very long processing times, generally in the range of one hour.

\section{ACKNOWLEDGMENTS}

We thank colleagues who assisted us with these analyses. Professor John Sader provided an analysis of the onset of mechanical nonlinearity in cantilevers. Jihun Kim performed simulations on bias-induced heating in fluid-filled microchannel resonators. Dr. Igor Bargatin provided valuable discussions on the optimization of thermoelastic actuation and mass detection. Finally we thank Drs. B. W. Axelrod and $X$. L. Feng for their critical review of the manuscript. We gratefully acknowledge support from DARPA under Grant No. HR001106.

${ }^{1}$ K. L. Ekinci, Y. T. Yang, and M. L. Roukes, J. Appl. Phys. 95, 2682 (2004)

${ }^{2}$ B.Ilic, H. G. Craighead, S. Krylov, W. Senaratne, C. Ober, and P. Neuzil, J. Appl. Phys. 95, 3694 (2004)

${ }^{3}$ Y. T. Yang, C. Callegari, X. L. Feng, K. L. Ekinci, and M. L. Roukes, Nano Lett. 6, 583 (2006)

${ }^{4}$ A. K. Naik, M. S. Hanay, W. K. Hiebert, X. L. Feng, and M. L. Roukes, Nat. Nanotechnol. 4, 445 (2009)

${ }^{5}$ J. L. Arlett, M. R. Paul, J. E. Solomon, M. C. Cross, S. E. Fraser, and M. L. Roukes, Lect. Notes Phys. 711, 241 (2007)

${ }^{6}$ M. B. Viani, T. E. Schaffer, A. Chand, M. Rief, H. E. Gaub, and P. K. Hansma, J. Appl. Phys. 86, 2258 (1999)

${ }^{7}$ T. P. Burg and M. L. Manalis, Appl. Phys. Lett. 83, 2698 (2003)

${ }^{8}$ J. Lee, W. Shen, K. Payer, T. P. Burg, and S. R. Manalis, Nano Lett. 10, 2537 (2010).

${ }^{9}$ T. P. Burg, A. R. Mirza, N. Milovic, C. H. Tsau, G. A. Popescu, J. S. Foster, and S. R. Manalis, J. Microelectromech. Syst. 15, 1466 (2006)

${ }^{10}$ Burg et al., Nature (London) 446, 1066 (2007)

${ }^{11}$ T. M. Squires, R. J. Messinger, and S. R. Manalis, Nat. Biotechnol. 26, 417 (2008).

${ }^{12}$ P. E. Sheehan and L. J. Whitman, Nano Lett. 5, 803 (2005).

${ }^{13}$ J. E. Solomon and M. Paul, Biophys. J. 90, 1842 (2006).

${ }^{14}$ P. R. Nair and A. A. Muhammad, Nano Lett. 8, 1281 (2008).

${ }^{15}$ K. L. Ekinci, Y. T. Yang, and M. L. Roukes, Appl. Phys. Lett. 95, 2682 (2004).

${ }^{16}$ S. Son, W. H. Grover, T. P. Burg, and S. Manalis, Anal. Chem. 80, 4757 (2008).

${ }^{17}$ P. Enoksson, G. Stemme, and E. Stemme, Sens. Actuators, A 54, 558 (1996).

${ }^{18}$ M. G. von Muhlen, N. D. Brault, S. M. Knudsen, S. Jiang, and S. R. Manalis, Anal. Chem. 82, 1905 (2010).

${ }^{19}$ R. Chunara, M. Godin, S. M. Knudsen, and S. R. Manalis, Appl. Phys. Lett. 91, 193902 (2007).

${ }^{20}$ M. Godin, A. K. Bryan, T. P. Burg, K. Babcock, and S. R. Manalis, Appl. Phys. Lett. 91, 123121 (2007)

${ }^{21}$ V. A. Van Eysden and J. E. Sader, J. Appl. Phys. 101, 044908 (2007).
${ }^{22}$ T. Braun, V. Barwich, M. K. Ghatkesar, A. H. Bredekamp, C. Gerber, M. Hegner, and H. P. Lang, Phys. Rev. E 72, 031907 (2005).

${ }^{23}$ M. K. Ghatkesar, V. Barwich, T. Braun, J. P. Ramseyer, C. Gerber, M. Hegner, H. P. Lang, U. Drechsler, and M. Despont, Nanotechnology 18, 445502 (2007)

${ }^{24}$ P. Gallay, S. Swingler, J. Song, F. Bushman, and D. Trono, Cell 83, 569 (1995).

${ }^{25}$ K. Y. Yasumura, T. D. Stowe, E. M. Chow, T. Pfafman, T. W. Kenny, B. C. Stipe, and D. Rugar, J. Microelectromech. Syst. 9, 117 (2000).

${ }^{26}$ I. Bargatin, I. Kokinsky, and M. L. Roukes, Appl. Phys. Lett. 90, 093116 (2007).

${ }^{27}$ M. Li, H. X. Tang, and M. L. Roukes, Nat. Nanotechnol. 2, 114 (2007).

${ }^{28}$ The Alliance for Nanosystems VLSI, www.nanovlsi.org, 2008 (unpublished).

${ }^{29}$ R. A. Barton, B. Ilic, S. S. Verbridge, B. R. Ciriany, J. M. Parpia, and H. G. Craighead, Nano Lett. 10, 2058 (2010).

${ }^{30}$ G. Piazza, P. J. Stephanou, and A. P. Pisano, J. Microelectromech. Syst. 15, 1406 (2006).

${ }^{31}$ K. S. Hwang, J. H. Lee, J. Park, D. S. Yoon, J. H. Park, and T. S. Kim, Lab Chip 4, 547 (2004).

${ }^{32}$ R. B. Karabalin, M. H. Matheny, X. L. Feng, G. Le Rhun, C. Marcoux, S. Hentz, P. Andreucci, and M. L. Roukes, Appl. Phys. Lett. 95, 103111 (2009).

${ }^{33}$ K. L. Ekinci, Small 1, 786 (2005).

${ }^{34}$ J. L. Arlett, E. B. Myers, and M. L. Roukes, "Fluidic micro and nanomechanical biosensors," Nat. Nanotechnol. (to be published).

${ }^{35}$ J. E. Sader, "Nonlinear response of cantilever and doubly-clamped beams," Nano Lett. (to be published).

${ }^{36}$ A. N. Cleland and M. L. Roukes, J. Appl. Phys. 92, 2758 (2002).

${ }^{37}$ T. R. Albrecht, P. Grütter, D. Horne, and D. Rugar, J. Appl. Phys. 69, 668 (1991).

${ }^{38}$ X. L. Feng, C. J. White, A. Hajimiri, and M. L. Roukes, Nat. Nanotechnol. 3, 342 (2008).

${ }^{39}$ I. Bargatin, and M. L. Roukes, Finite-element simulation of thermoelastic actuation of NEMS (unpublished).

${ }^{40}$ The formula used here is that for flow-through a channel with a rectangular cross-section. See, for example, C. Pozrikidis, Introduction to Theoretical and Computational Fluid Dynamics (Oxford University Press, New York, 1997), pp. 180-187.

${ }^{41}$ S. C. Masmanidis, R. B. Karabalin, I. De Vlaminick, G. Borghs, M. R. Freeman, and M. L. Roukes, Science 317, 780 (2007).

${ }^{42}$ Z. Shen, W. Y. Shih, and W.-H. Shih, Appl. Phys. Lett. 89, 023506 (2006).

${ }^{43}$ J. E. Sader, personal communication (21, August, 2008).

${ }^{44}$ J. R. Heath and M. E. Davis, Annu. Rev. Med. 59, 251 (2008).

${ }^{45}$ In many SMR applications all four sidewalls are functionalized. Here, for simplicity, we consider only the top and bottom surface (representing $85 \%$ of the total device surface area) to be functionalized.

${ }^{46}$ The diffusion constant of an HIV virion was estimated using the EinsteinStokes equation for a spherical particle of $100 \mathrm{~nm}$ diameter in water.

${ }^{47}$ R. C. Ackerberg, R. D. Patel, and S. K. Gupta, J. Fluid Mech. 86, 49 (1978).

${ }^{48} \mathrm{~J}$. Newman, in Electroanalytical Chemistry, edited by A. Bard (Dekker, New York, 1973), Vol. 6, p. 279.

${ }^{49}$ G. Wu, R. H. Dam, K. M. Hansen, T. Thundat, R. J. Cote, and A. Majumdar, Nat. Biotechnol. 19, 856 (2001).

${ }^{50}$ X. Huang, C. T. Knoll, G. Frey, M. Hazegh-Azam, A. H. Tashjian, Jr., L. Hedstrom, and R. H. Abeles, Biochemistry 40, 11734 (2001).

${ }^{51}$ P. S. Katsamba, I. Navratilova, M. Calderon-Cacia, L. Fan, K. Thornton, M. Zhu, T. V. Bos, C. Forte, D. Friend, I. Laird-Offringa G. Tavares, J. Whatley, E. Shi, A. Widom, K. C. Lindquist, S. Klakamp, A. Drake, D. Bohmann, M. Roell, L. Rose, J. Dorocke, B. Roth, B. Luginbühl, and D. G. Myszka, Anal. Biochem. 352, 208 (2006).

${ }^{52}$ D. G. Myszka, T. A. Morton, M. L. Doyle, and I. M. Chaiken, Biophys. Chem. 64, 127 (1997).

${ }^{53}$ P. Peluso, D. S. Wilson, D. Do, H. Tran, M. Venkatasubbaiah, D. Quincy, B. Heidecker, K. Poindexter, N. Tolani, M. Phelan, K. Witte, L. S. Jung, P. Wagner, and S. Nock, Anal. Biochem. 312, 113 (2003). 